\title{
Quantum sign permutation polytopes
}

\author{
Colin Wilmott, Hermann Kampermann and Dagmar Bruß \\ Institut für Theortische Physik III, Heinrich-Heine-Universität, Düsseldorf, Germany \\ E-mail: wilmott@thphy.uni-duesseldorf.de
}

\begin{abstract}
Convex polytopes are convex hulls of point sets in the $n$-dimensional space $\mathbb{E}^{n}$ that generalize 2-dimensional convex polygons and 3-dimensional convex polyhedra. We concentrate on the class of $n$-dimensional polytopes in $\mathbb{E}^{n}$ called sign permutation polytopes. We characterize sign permutation polytopes before relating their construction to constructions over the space of quantum density matrices. Finally, we consider the problem of state identification and show how sign permutation polytopes may be useful in addressing issues of robustness.

PACS numbers: 02.40.Dr, 02.40.Ft
\end{abstract}

\section{Introduction}

From the Platonic solids to $n$-cubes, $n$-cross-polytopes and regular $n$-simplices, convex polytopes as geometric objects have been a source of study since antiquity. Indeed, 'the Universe cannot be read until we have learned the language in which it is written. It is written in mathematical language, and the letters are triangles, circles and other geometrical figures' - Galileo. A celebrated feature of these convex geometrical figures has been the symmetry they possess. In micro-biology where objects use the efficiency of symmetry to propagate themselves to the study of genetics where symmetry communicates genetic information, symmetry has been a fundamental concept of science and has provided an understanding for many of Nature's phenomena. But perhaps the most important class of symmetric polytopes is the class of regular convex polytopes in Euclidean spaces.

In this paper, we concern ourselves with the class of $n$-dimensional convex polytopes in $\mathbb{E}^{n}$ called sign permutation polytopes. We show that regular convex polytopes in Euclidean space can be derived from such polytopes, and we show that their characterization can be admitted by the partial order of weak majorization. The outline of this paper is as follows. Section 2 provides an introduction to material from discrete geometry which serves as a basis for our study. Section 3 introduces a theorem of Rado (Rado (1952)) and motivates the issue of $n$-dimensional polytopes of degree $n$ in the $n$-dimensional space $\mathbb{E}^{n}$ of section 4 . Section 5 relates the construction of sign permutation polytopes to the space of density matrices in quantum mechanics, before 
finally illustrating how sign permutation polytopes may be useful in addressing the problem of robustness of state identification.

\section{Preliminaries}

\subsection{Convex sets \& combinations}

Let $n \in \mathbb{N}$ and denote by $\mathbb{E}^{n}$ the $n$-dimensional Euclidean space, with origin $o$, scalar product $\langle\cdot, \cdot\rangle$ and induced norm $\|\cdot\|$. Let $a=\left(\alpha_{1}, \alpha_{2}, \ldots, \alpha_{n}\right) \in \mathbb{E}^{n}$ be an $n$-tuple of real numbers. We say $a \in \mathbb{E}^{n}$ is a linear combination of $a_{1}, \ldots, a_{m} \in \mathbb{E}^{n}$ if $a=\sum_{i=1}^{m} \lambda_{i} a_{i}$ for suitable scalars $\lambda_{i} \in \mathbb{R}$. If such scalars $\lambda_{i} \in \mathbb{R}, i=1, \ldots, m$, exist with $\sum_{i=1}^{m} \lambda_{i}=1$ then we say $a$ is an affine combination of $a_{1}, \ldots, a_{m} \in \mathbb{E}^{n}$. The set of points $a_{1}, \ldots, a_{m} \in \mathbb{E}^{n}$ is said to be affinely independent if a linear combination $\sum_{i=1}^{m} \lambda_{i} a_{i}$ with $\sum_{i=1}^{m} \lambda_{i}=0$ can only have the value $o$ when $\lambda_{i}=0$ for $i=1, \ldots, m$. For $A \subset \mathbb{E}^{n}$, the set of all affine combinations of points of $A$ is called the affine hull of $A$ and is denoted by aff $A$. If $a=\sum_{i=1}^{m} \lambda_{i} a_{i}$ for non-negative scalars $\lambda_{i} \in \mathbb{R}$ and $\sum_{i=1}^{m} \lambda_{i}=1$ then we say $a \in \mathbb{E}^{n}$ is a convex combination of $a_{1}, \ldots, a_{m} \in \mathbb{E}^{n}$. For $A \subset \mathbb{E}^{n}$, the set of all convex combinations of any finitely many points of $A$ is called the convex hull of $A$ and is denoted by $\operatorname{conv} A$. By $H_{a, \beta}$, we mean the hyperplane given by the linear equation of the form $\langle a, x\rangle=\beta$. Consequently, the hyperplane $H_{a, \beta}$ can be expressed as the set $\left\{x \in \mathbb{E}^{n} \mid\langle a, x\rangle=\beta\right\}$ where $a \in \mathbb{E}^{n} \backslash\{o\}$ and $\beta \in \mathbb{R}$. A closed half-space of $\mathbb{E}^{n}$ is given by the set $\left\{x \in \mathbb{E}^{n} \mid\langle a, x\rangle \geq \beta\right\}, a \in \mathbb{E}^{n} \backslash\{o\}$ and $\beta \in \mathbb{R}$, where its boundary is the hyperplane $\left\{x \in \mathbb{E}^{n} \mid\langle a, x\rangle=\beta\right\}, a \in \mathbb{E}^{n} \backslash\{o\}$ and $\beta \in \mathbb{R}$. For convex sets $A, B \subset \mathbb{E}^{n}$ satisfying $A \cap B=\emptyset$, the Hahn-Banach separation theorem (Bishop and Bridges (1985)) states that there exists a unit vector $u \in \mathbb{E}^{n}$ and a scalar $\beta \in \mathbb{R}$ such that for all $a \in A$ we have $\langle u, a\rangle \geq \beta$, while for all $a \in B$ we have $\langle u, a\rangle \leq \beta$.

\subsection{The symmetric group \& majorization}

Let $S_{n}$ denote the symmetric group of degree $n$ defined as the group of all permutations of the integers $1,2, \ldots, n$. Denote by $\pi(a)=\left(\alpha_{\pi(1)}, \ldots, \alpha_{\pi(n)}\right)$, the permutation of $a \in \mathbb{E}^{n}$ by $\pi \in S_{n}$. Let $a^{*}=\left(\alpha_{1}^{*}, \ldots, \alpha_{n}^{*}\right) \in \mathbb{E}^{n}$ be the point whose components are those of $a$ arranged in non-increasing order of magnitude; $\alpha_{1}^{*} \geq \ldots \geq \alpha_{n}^{*}$ and $\alpha_{k}^{*}=\alpha_{\pi(k)}$, for $k=1, \ldots, n$ and $\pi \in S_{n}$. Let $b^{*}=\left(\beta_{1}^{*}, \ldots, \beta_{n}^{*}\right) \in \mathbb{E}^{n}$ be defined analogously. If the relations

$$
\sum_{k \leq l} \alpha_{k}^{*} \leq \sum_{k \leq l} \beta_{k}^{*}
$$

hold, for $1 \leq l \leq n$, with equality for $l=n$, we say $a$ is majorized by $b$ and write $a \prec b$ (Marshall and Olkin (1979)). For $a, b \in \mathbb{E}^{n}$, we have it that

$$
a+b \prec a^{*}+b^{*}
$$

since there exists a permutation $\pi \in S_{n}$ such that $\alpha_{\pi(1)}+\beta_{\pi(1)} \geq \alpha_{\pi(2)}+\beta_{\pi(2)} \geq \ldots \geq$ $\alpha_{\pi(n)}+\beta_{\pi(n)}$ and $\sum_{k \leq l}\left(\alpha_{\pi(k)}+\beta_{\pi(k)}\right) \leq \sum_{k \leq l}\left(\alpha_{k}^{*}+\beta_{k}^{*}\right)$, for $1 \leq l \leq n$ with equality 
for $l=n$. Consider the set of points $a_{1}, a_{2}, \ldots, a_{m} \in \mathbb{E}^{n} ; a_{i}=\left(\alpha_{i 1}, \alpha_{i 2}, \ldots, \alpha_{i n}\right)$, $i=1,2, \ldots, m$. Following from (2), we have it that $a_{1}+a_{2}+\ldots+a_{m} \prec a_{1}^{*}+a_{2}^{*}+\ldots+a_{m}^{*}$. Finally, if $a_{i} \prec b, i=1, \ldots, m$, then $\sum_{i \leq m} \lambda_{i} a_{i} \prec b$ for non-negative scalars $\lambda_{i}$ and $\sum_{i \leq m} \lambda_{i}=1$.

\subsection{Convex polytopes}

Convex polytopes are non-empty compact convex subsets of Euclidean space described by a finite point set. Although the concept of convexity is elementary, polytopes possessing this property yield geometric bodies with rich structure. Two-dimensional convex polytopes are called convex polygons. The equilateral triangle constructed by Euclid in his first proposition is such an example. Three-dimensional convex polytopes are called convex polyhedrons and examples include the Platonic solids (Coxeter (1969)). Interestingly, while the polyhedron has eluded definition over the centuries, the theory of convex polyhedrons and polytopes is well understood and contributes to practically significant areas of combinatorial optimization and computational geometry. To see this, consider the permutahedron defined by taking the convex hull of all vectors that are obtained by permuting the coordinates of the vector $(1,2, \ldots, n)$. A feature of the permutahedron is that the vertices are identified with the permutations of the symmetric group of degree $n$ in such a way that two vertices are connected if and only if the corresponding permutations differ by an adjacent transposition (Gaiha and Gupta (1977)).

A convex polytope is the convex hull of a non-empty finite set $A=$ $\left\{a_{1}, a_{2}, \ldots, a_{m}\right\} \subset \mathbb{E}^{n}$. In particular, the $\mathcal{V}$-polytope is the set of points describing such a polytope in terms of its vertices and is given as

$$
\left\{\sum_{i=1}^{m} \lambda_{i} a_{i} \mid \lambda_{i} \geq 0, \sum_{i=1}^{m} \lambda_{i}=1\right\} .
$$

On the other hand, a convex polytope may be described as the bounded solutions set of a finite system of half-spaces in $\mathbb{E}^{n}$. In this instance, we say such a polytope is a $\mathcal{H}$-polytope and is described as

$$
\left\{x \in \mathbb{E}^{n} \mid\left\langle a_{i}, x\right\rangle \leq \beta_{i} \text { for } 1 \leq i \leq m\right\},
$$

for $a_{i} \in \mathbb{E}^{n}$ and $\beta_{i} \in \mathbb{R}$. A basic result of convex polytopes maintains that we may regard a convex polytope as a bounded solution set of finitely many closed half-spaces in $\mathbb{E}^{n}$ or as the convex hull of a non-empty finite set $A \subset \mathbb{E}^{n}$. Remarkably, while the descriptions of a convex polytope as that of a $\mathcal{H}$-polytope or as a $\mathcal{V}$-polytope are equivalent, the computational complexity associated with describing each differs in the extreme. For instance, the $n$-dimensional cross-polytope as a $\mathcal{V}$-polytope is defined as the convex hull of the set of $2 n$ points

$$
\gamma_{n}=\operatorname{conv}\left\{\pi( \pm 1,0, \ldots, 0), \pi \in S_{n}\right\},
$$

whereas, as a $\mathcal{H}$-polytope, the $n$-cross-polytope is described by $2^{n}$ half-spaces

$$
\gamma_{n}=\left\{x \in \mathbb{E}^{n} \mid\langle a, x\rangle \leq 1\right\}
$$


where $a$ runs through all vectors in $\{-1,1\}^{n}$. The surface of octahedron, a 3-crosspolytope, consists of six 0-dimensional faces called vertices, twelve 1-dimensional faces called edges and eight 2-dimensional faces called facets. Generally, the faces of an $n$-dimensional convex polytope have dimensions $-1,0,1, \ldots, n$ with -1 denoting the dimension of the empty set with the face of dimension $j$ being called a $j$-face. Finally, for $A \subset \mathbb{E}^{n}$, a convex polytope is called a $k$-polytope if $\operatorname{dim}$ conv $A=k$. This implies that some $(k+1)$-subset of $A$ is affinely independent but no such $(k+2)$-subset is affinely independent (Ziegler (1995)).

\section{A note on Rado's theorem}

Rado (1952) characterized the convex hull of the set of all permutations of any real $n$-tuple in terms of the Hardy-Littlewood-Pólya partial order relation (see Hardy et al (1934), p.45) for real $n$-tuples. We now state Rado's theorem and discuss the nature of the convex set established by the theorem.

Theorem 3.1 (Rado (1952)) Let $a \in \mathbb{E}^{n}$ and $\mathcal{V}_{a}$ be the convex hull of the set of permutations of $a$. Then $x \in \mathcal{V}_{a}$ if and only if $x \prec a$.

The polytope of Rado (1952) is the $(n-1)$-dimensional permutahedron of degree $n$ embedded in the $n$-dimensional space $\mathbb{E}^{n}$. Since the vertices are obtained by permuting the integers $(1,2, \ldots, n)$, this permutahedron of degree $n$ lies entirely in an $(n-1)$ dimensional hyperplane consisting of all points whose coordinates sum to the integer $1+2+\ldots+n=n(n+1) / 2$. For the application in quantum state identification, we concern ourselves with the construction of $n$-dimensional polytopes of degree $n$ in the $n$-dimensional space $\mathbb{E}^{n}$ that exploit the key feature of Rado's theorem. We now show that a necessary and sufficient condition for such polytopes requires a set of $(n-1)$ dimensional bounding facets to contain points whose coordinate sums differ.

Proposition 3.2 Let $a, b \in \mathbb{E}^{n}$. Let $\mathcal{V}_{a}$ and $\mathcal{V}_{b}$ denote the convex hull of the set of permutations of $a$ and $b$, respectively. Then $\mathcal{V}_{a} \cap \mathcal{V}_{b}=\emptyset$ if and only if $\sum_{k \leq n} \alpha_{k} \neq$ $\sum_{k \leq n} \beta_{k}$

To establish proposition 3.2, we make use of the following results. Let $A=$ $\operatorname{conv}\left\{a_{1}, \ldots, a_{m}\right\} \subset \mathbb{E}^{n}$ be a non-empty convex set. By the relative interior of $A$, relint $A$, we mean those points $a \in \mathbb{E}^{n}$ for which $a=\sum_{i=1}^{m} \lambda_{i} a_{i}$ with $\lambda_{i}>0$, for $i=1, \ldots, k$, and $\sum_{i=1}^{m} \lambda_{i}=1$.

Lemma 3.3 (Grünbaum (2003)) Let $A, B \subset \mathbb{E}^{n}$ be nonempty convex sets. Then $A$ and $B$ can be properly separated if and only if

$$
\operatorname{relint} A \cap \operatorname{relint} B=\emptyset \text {. }
$$

Lemma 3.4 If $a, b \in \mathbb{E}^{n}$ such that $\sum_{k \leq n} \alpha_{k}=\sum_{k \leq n} \beta_{k}$, then $\mathcal{V}_{a}$ and $\mathcal{V}_{b}$ can not be properly separated by a hyperplane $H_{u, \alpha}$,

$$
H_{u, \alpha}=\left\{z \in \mathbb{E}^{n} \mid\langle z, u\rangle=\alpha\right\}
$$




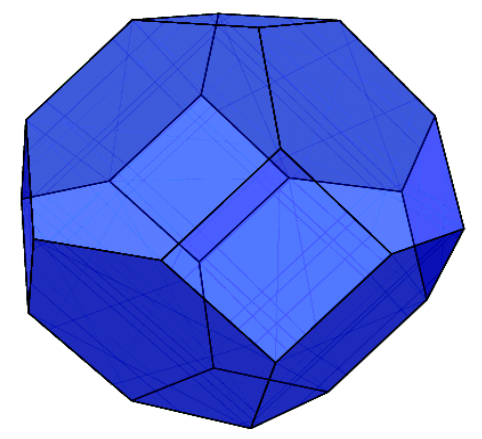

Figure 1. Truncated octahedron: sign permutation polytope with basis vector $a=(0,1,2)$ centered at the origin. The truncated octahedron is an Archimedean solid consisting of 8 regular hexagonal facets, 6 square facets, 36 edges and 24 vertices.

for $u \in \mathbb{E}^{n} \backslash\{o\}$, and $\alpha \in \mathbb{R}$.

Proof. See Appendix A. Proof of proposition 3.2.

Let us suppose that $\sum_{k \leq n} \alpha_{k}=\sum_{k \leq n} \beta_{k}$. By lemma 3.4. the convex hulls $\mathcal{V}_{a}$ and $\mathcal{V}_{b}$ can not be properly separated. Consequently, by lemma 3.3, we have $\mathcal{V}_{a} \cap \mathcal{V}_{b} \neq \emptyset$ and the implication follows. On the other hand, suppose $\mathcal{V}_{a} \cap \mathcal{V}_{b} \neq \emptyset$. Hence there exists $x \in \mathcal{V}_{a} \cap \mathcal{V}_{b}$, and therefore numbers $\lambda_{i} \geq 0, \mu_{i} \geq 0$ satisfying $\sum_{i \leq m} \lambda_{i}=\sum_{i \leq m^{\prime}} \mu_{i}=1$ such that $x=\sum_{i \leq m} \lambda_{i} a_{i}$ and $x=\sum_{i \leq m^{\prime}} \mu_{i} b_{i}$. For $l \leq n$ and $x=\left(x_{1}, x_{2}, \ldots, x_{n}\right) \in \mathbb{E}^{n}$, we have it that

$$
\sum_{k \leq l} x_{k}=\sum_{k \leq l} \sum_{i \leq m} \lambda_{i} \alpha_{i k}=\sum_{i \leq m} \lambda_{i} \sum_{k \leq l} \alpha_{i k} \leq \sum_{i \leq m} \lambda_{i} \sum_{k \leq l} \alpha_{k}^{*}=\sum_{k \leq l} \alpha_{k}^{*},
$$

with equality for $l=n$. Similarly, for $l \leq n$, we have it that

$$
\sum_{k \leq l} x_{k}=\sum_{k \leq l} \sum_{i \leq m^{\prime}} \mu_{i} \beta_{i k}=\sum_{i \leq m^{\prime}} \mu_{i} \sum_{k \leq l} \beta_{i k} \leq \sum_{i \leq m^{\prime}} \mu_{i} \sum_{k \leq l} \beta_{k}^{*}=\sum_{k \leq l} \beta_{k}^{*},
$$

with equality for $l=n$. The implication follows, and this completes the proof.

While proposition 3.2 illustrates a necessary and sufficient criterion to construct an $n$-dimensional polytope if given an initial $(n-1)$-dimensional permutahedron, it suggests a generalization of Rado's theorem is not readily available since the inclusion of an arbitrary point does not preserve the majorization criterion. For example, consider $a, b \in \mathbb{E}^{n}$ and further consider the polytope described by taking the convex hull of $\mathcal{V}_{a}$ and $\mathcal{V}_{b}$. Let us denote such a polytope by $\operatorname{conv}\left\{\mathcal{V}_{a}, \mathcal{V}_{b}\right\}$. For some $x \in \mathcal{V}_{a}$ and $y \in \mathcal{V}_{b}$, the question of whether a point $z \in \mathbb{E}^{n}$ is contained in the polytope $\operatorname{conv}\left\{\mathcal{V}_{a}, \mathcal{V}_{b}\right\}$ can be verified by showing that there exists a suitable $\lambda \in \mathbb{R}, 0 \leq \lambda \leq 1$, such that $z=\lambda x+(1-\lambda) y$. Consequently, we can demonstrate that $z \prec \lambda a+(1-\lambda) b$, and, hence, $z \in \operatorname{conv}\left\{\mathcal{V}_{a}, \mathcal{V}_{b}\right\}$ but only at the insistence of a suitably chosen scalar $\lambda \in \mathbb{R}$. 


\section{Sign permutation polytopes}

Let us instead consider the task of constructing a polytope whose vertices are not arbitrary $n$-tuples in $\mathbb{E}^{n}$. We adapt the theorem of Rado (1952) to construct an $n$ dimensional polytope of degree $n$ in $\mathbb{E}^{n}$ with vertices given by sign permutation points of $\mathbb{E}^{n}$ and an interior that admits a characterization with respect to the partial order of weak majorization.

Consider the convex polytope whose vertices consist of the set of sign permutations on the integers $(1,2, \ldots, n)$ defined as the set of all permutations of $( \pm 1, \pm 2, \ldots, \pm n)$. We call such a polytope consisting of $2^{n} n$ ! vertices a sign permutation polytope, see figure 1. The sign permutation polytope is $n$-dimensional convex polytope of degree $n$ in the $n$-dimensional space $\mathbb{E}^{n}$. More generally, denote by $\mathcal{V}_{ \pm \pi(a)}$ the sign permutation polytope given as the convex hull of componentwise sign permutation changes of $a \in \mathbb{E}^{n}$;

$$
\mathcal{V}_{ \pm \pi(a)}=\operatorname{conv}\left\{\left( \pm \alpha_{\pi(1)}, \pm \alpha_{\pi(2)}, \ldots, \pm \alpha_{\pi(n)}\right), \pi \in S_{n}\right\}
$$

Let $a^{*} \in \mathbb{E}^{n}$ denote again that vector whose components are those of $a$ arranged in non-increasing order of magnitude. Let $b \in \mathbb{E}^{n}$ be defined analogously. If the relations

$$
\sum_{k \leq l} \alpha_{k}^{*} \leq \sum_{k \leq l} \beta_{k}^{*}
$$

hold, for $1 \leq l \leq n-1$, and

$$
\sum_{k \leq n} \alpha_{k}^{*}<\sum_{k \leq n} \beta_{k}^{*}
$$

we then say $a$ is weakly majorized by $b$, and write $a \prec_{w} b$. Let $\mathbb{E}_{+}^{n}$ denote the set $\mathbb{E}_{+}^{n}=\left\{\left(\alpha_{1}, \ldots, \alpha_{n}\right) \mid \alpha_{i} \geq 0, i=1, \ldots, n\right\}$.

Corollary 4.1 Let $a, x \in \mathbb{E}_{+}^{n}$. Then $x \in \mathcal{V}_{ \pm \pi(a)}$ if and only if $x \prec_{w} a$.

Proof. If $x \in \mathcal{V}_{ \pm \pi(a)}$, then there are numbers $\lambda_{i}$ and vectors $a_{i}, i=1,2, \ldots, m$, for $m \leq 2^{n} n$ ! such that $a_{i} \prec_{w} a, \lambda_{i} \geq 0, \sum_{i \leq m} \lambda_{i}=1$ and $x=\sum_{i \leq m} \lambda_{i} a_{i}$. Then since $a_{i} \prec_{w} a$, we have it that $x=\sum_{i \leq m} \lambda_{i} a_{i} \prec_{w} \sum_{i \leq m} \lambda_{i} a=a$ for $\lambda_{i} \geq 0, i=1,2, \ldots, m$, and $\sum_{i \leq m} \lambda_{i}=1$. On the other hand, suppose $x \prec_{w} a$ while $x \notin \mathcal{V}_{ \pm \pi(a)}$. Using the Hahn-Banach separation argument of Rado (1952), the reverse implication is readily established, and the result follows.

We make note of the following relationship between the convex hulls of $\mathcal{V}_{ \pm \pi(a)}$ and $\mathcal{V}_{ \pm \pi(x)}$.

Corollary 4.2 Let $a, x \in \mathbb{E}_{+}^{n}$. Let $\mathcal{V}_{ \pm \pi(a)}$ and $\mathcal{V}_{ \pm \pi(x)}$ denote the convex hull of sign permutations of $a, x \in \mathbb{E}_{+}^{n}$, respectively. Suppose $x \prec_{w}$ a. Then $\mathcal{V}_{ \pm \pi(x)} \subseteq \mathcal{V}_{ \pm \pi(a)}$.

Proof. Given $a, x \in \mathbb{E}_{+}^{n}$ with $x \prec_{w} a$, we have it that $\pm \pi(x) \prec_{w} x$ for $\pi \in S_{n}$. Noting that $\prec_{w}$ is a partial order then transitivity of $\prec_{w}$ establishes $\pm \pi(x) \prec_{w} \pm \pi(a)$, and the result follows.

Remark 4.3 We note that Mirsky (1959) has constructed a polytope similar to the polytope of corollary 4.1 restricted to the positive orthant of n-dimensional Euclidean space. Markus (1964) characterized the boundary of the sign permutation polytope. 
A regular polytope generalizes the Platonic solids to arbitrary dimensions. For the $n$-dimensional spaces $\mathbb{E}^{n}$ with $n \geq 5$, there are only three regular convex polytopes (Coxeter (1948)). These are the $n$-dimensional regular simplex, the $n$-dimensional crosspolytope and the $n$-dimensional cube. For $a=(\alpha, 0, \ldots, 0) \in \mathbb{E}^{n}$, the sign permutation polytope $\mathcal{V}_{ \pm \pi(a)}$ degenerates to the class of cross-polytopes $\beta_{n}$. The cross-polytope $\beta_{n}$ can be described in terms of the $2 n$ vertex set $\left\{\pi( \pm \alpha, 0, \ldots, 0), \pi \in S_{n}\right\}$, or, equally, in terms of the $2^{n}$ half spaces $\gamma_{n}=\left\{x \in \mathbb{E}^{n} \mid\langle a, x\rangle \leq 1\right\}$, where $a$ runs through all vectors in $\{-1,1\}^{n}$. For $a=(\alpha, \alpha, \ldots, \alpha) \in \mathbb{E}^{n}$, the sign permutation polytope $\mathcal{V}_{ \pm \pi(a)}$ coincides with the $n$-cube $\gamma_{n}$ which can be evaluated either in terms of the $2 n$ halfspaces $\left\{x \in \mathbb{E}^{n} \mid-\alpha \leq x_{k} \leq \alpha\right.$, for $\left.1 \leq k \leq n\right\}$, or, equivalently, with respect to the $2^{n}$ vertex set $\{( \pm \alpha, \pm \alpha, \ldots, \pm \alpha)\}$. More generally, if we consider the $n$-tuple of real coefficients $a=\left(\alpha_{1}, \ldots, \alpha_{m}, 0_{1}, \ldots, 0_{n-m}\right) \in \mathbb{E}^{n}$ consisting of $k \leq n$ distinct coefficients of multiplicity $m_{i}, 1 \leq i \leq k$, then the number of distinct sign permutations, i.e. vertices, in the set $\left\{\pi\left( \pm \alpha_{1}, \ldots, \pm \alpha_{m}, 0_{1}, \ldots, 0_{n-m}\right), \pi \in S_{n}\right\}$ is

$$
\frac{2^{n} n !}{2^{n-m} m_{1} ! \ldots m_{k-1} !(n-m) !} .
$$

Consequently, we have it that the sign permutation polytope $\mathcal{V}_{ \pm \pi(a)}$ given by

$$
\operatorname{conv}\left\{\pi\left( \pm \alpha_{1}, \ldots, \pm \alpha_{m}, 0_{1}, \ldots, 0_{n-m}\right), \pi \in S_{n}\right\}
$$

has a vertex set of order $2^{m} n ! /\left(m_{1} ! \ldots m_{k-1} !(n-m) !\right)$. We see again that for $m=1$, the sign permutation polytope $\mathcal{V}_{ \pm \pi(a)}, a=\left(\alpha_{1}, \ldots, \alpha_{m}, 0_{1}, \ldots, 0_{n-m}\right) \in \mathbb{E}^{n}, m \leq n$, describes the $n$-dimensional cross-polytope $\beta_{n}$, and for $m=n$ and $k=n$, the sign permutation polytope coincides with the $n$-dimensional cube $\gamma_{n}$. For $a=(\alpha, 0, \ldots, 0) \in \mathbb{E}^{n+1}$, we note that the $n$-dimensional regular simplex $\alpha_{n}$ is a permutation polytope of degree $n+1$ defined as the convex hull of all permutations of the vector $a \in \mathbb{E}^{n+1}$.

Finally, for what follows, we derive the volume and insphere radius for the $n$-crosspolytope.

Lemma 4.4 Let $\alpha \geq 0$. The volume of the $n$-dimensional cross-polytope $\operatorname{conv}\left\{\pi( \pm \alpha, 0, \ldots, 0), \pi \in S_{n}\right\}$ of edge length $\sqrt{2} \alpha$ is $(2 \alpha)^{n} /(n)$ !.

The cross-polytope $\beta_{n}$ is a composition of $2^{n}$ convex regions with each region corresponding to an orthant of $\beta_{n}$. The convex region determined by the positive orthant of $\beta_{n}$ is an $n$-dimensional cone of height $\alpha$. In $\mathbb{E}^{n}$, the volume of the cone of height $h$ over an $(n-1)$-dimensional base of volume $B$ is given as $B h / n$. By induction, the volume of the cone in the positive orthant of $\beta_{n}$ is $\alpha^{n} / n$ !. Consequently, the volume of $\beta_{n}$ is $(2 \alpha)^{n} / n !$.

Next we consider the inscribed sphere of the cross-polytope.

Lemma 4.5 The insphere of the cross-polytope $\operatorname{conv}\left\{\pi( \pm \alpha, 0, \ldots, 0), \pi \in S_{n}\right\}, \alpha \geq 0$, of side length $\sqrt{2} \alpha$ has radius $r_{\mathrm{in}}=\alpha / \sqrt{n}$.

Proof. We maximize the $n$-dimensional sphere $\sum_{i=1}^{n} x_{i}{ }^{2}=r^{2}$ subject to the constraint $\sum_{i=1}^{n} x_{i}=\alpha$. The constraint $\sum_{i=1}^{n} x_{i}=\alpha$ represents the coordinate sum of the points 
on the facet of the cross-polytope in the positive orthant of $\mathbb{E}^{n}$. Let $L\left(x_{1}, x_{2}, \ldots, x_{n}\right)=$ $\sum_{i=1}^{n} x_{i}{ }^{2}-\lambda\left(\sum_{i=1}^{n} x_{i}-\alpha\right)$. By the method of Lagrange multipliers, we have it that $\nabla L\left(x_{1}, x_{2}, \ldots, x_{n}\right)=\left\{2 x_{i}-\lambda, i=1, \ldots, n\right\}$ with $\sum_{i=1}^{n} x_{i}=\alpha$. Solving the system of equations yields $x_{i}=\alpha / n$ for $i=1, \ldots, n$, and the result follows.

\section{Quantum sign permutation polytopes}

A fundamental topic of quantum information theory has been the characterization of entanglement amongst states of a quantum system. A natural question concerns the partitioning of the set of all states of some finite dimensional composite quantum system according to entanglement type. It has been shown that all bipartite entangled pure states are asymptotically equivalent, up to local operations and classical communication, to the Einstein-Podolsky-Rosen state (Bennett et al (1996)). For systems of three or more parties, there are several equivalence classes of different entanglement types (Vidal (2000)). We note the particular instance of three qubit systems in which Dür et al (2000) has shown that there are two equivalence classes possessing genuine tripartite entanglement.

In this article, we focus on the construction of sign permutations polytopes within the space of quantum states, paying attention to three-qubit systems. We demonstrate the construction of quantum sign permutation polytopes for both pure and mixed quantum states, and we utilize the weak majorization feature admitted by sign permutation polytopes to classify a certain subset of quantum states. The construction of sign permutation polytopes from section 4 can be translated to the quantum world as follows.

Let $d \in \mathbb{N}$ and let $\mathcal{H}$ denote the $d$-dimensional complex Hilbert space. Let $\mathcal{M}_{d}$ denote the set of states that coincide with the set of $d \times d$ complex Hermitian matrices with non-negative eigenvalues and unit trace which act on the Hilbert space $\mathcal{H}$;

$$
\mathcal{M}_{d}=\left\{\varrho \mid \varrho=\varrho^{\dagger} ; \varrho \geq 0 ; \operatorname{Tr} \varrho=1\right\}
$$

$\mathcal{M}_{d}$ is a $\left(d^{2}-1\right)$-real-dimensional convex set and the vertices of this set form a $2(d-1)$ dimensional subspace of the $\left(d^{2}-2\right)$-dimensional boundary $\partial \mathcal{M}_{d}$. The set of density matrices of $\mathcal{M}_{d}$ consists of pure and mixed states and the pure states correspond to the extreme points of this set. For $\varrho \in \mathcal{M}_{d}, \varrho$ is a pure state if and only if $\varrho^{2}=\varrho$ with $\varrho$ written as a rank one projector onto $\mathcal{H}$. Otherwise, $\varrho$ is a mixed state, and we write $\varrho$ as a convex combination of pure states. Noting that any general $n$-dimensional

affine space $\mathbb{A}$ is isomorphic the $n$-dimensional affine space $\mathbb{E}^{n}$ (Brøndsted (1983)), we put $n=d^{2}-1$, and introduce a correspondence between a state $\varrho_{a} \in \mathcal{M}_{d}$ and a point $a \in \mathbb{E}^{d^{2}-1}$ by 


$$
\begin{aligned}
& \mathcal{M}_{d} \rightarrow \mathbb{E}^{d^{2}-1} \\
& \varrho_{a}=\left(\begin{array}{ccc}
\alpha_{1} & \alpha_{2}+\alpha_{3} \iota & \ldots \\
\alpha_{2}-\alpha_{3} \iota & \alpha_{4} & \ldots \\
& & \ddots
\end{array}\right) \mapsto\left(\begin{array}{c}
\alpha_{1} \\
\alpha_{2} \\
\alpha_{3} \\
\alpha_{4} \\
\vdots
\end{array}\right)=a .
\end{aligned}
$$

To ensure that $n$-dimensional polytopes have non-zero volume in $\mathbb{E}^{n}$, we require that the dimension of the corresponding affine hull be $n+1$. This fact may be demonstrated by noting that affine independence of an $(n+1)$-family of points in $\mathbb{E}^{n}$ is established if and only if there is linear independence amongst an augmentation of the $(n+1)$-family of points in $\mathbb{E}^{n+1}$.

Lemma 5.1 (Brøndsted (1983)) Let $\left\{a_{i}\right\} \in \mathbb{E}^{n}, i=0, \ldots, n$, and let $\tau(a): \mathbb{E}^{n} \rightarrow \mathbb{E}^{n+1}$ be defined by $\tau(a)=(a, 1)$. The set $\left\{a_{i}\right\}, i=0, \ldots, n$, is affinely independent in $\mathbb{E}^{n}$ if and only if $\left\{\left(a_{i}, 1\right)\right\}, i=0, \ldots, n$, is linearly independent in $\mathbb{E}^{n+1}$.

Proof. Suppose that $\left\{a_{i}\right\}, i=0, \ldots, n$, is affinely independent in $\mathbb{E}^{n}$, i.e., $\sum_{i=0}^{n} \lambda_{i} a_{i}$ vanishes in $\mathbb{E}^{n}$ and $\sum_{i=0}^{n} \lambda_{i}$ vanishes in $\mathbb{R}$ only when $\lambda_{i}=0, i=0, \ldots, n$. In particular, we have $\left(\sum_{i=0}^{n} \lambda_{i} a_{i}, \sum_{i=0}^{n} \lambda_{i}\right)=\sum_{i=0}^{n} \lambda_{i}\left(a_{i}, 1\right)=\left(0_{\mathbb{E}^{n}}, 0_{\mathbb{R}}\right)=0_{\mathbb{E}^{n+1}}$ only when $\lambda_{i}=0$, $i=0, \ldots, n$. Consequently, $\left\{\left(a_{i}, 1\right)\right\}, i=0, \ldots, n$, is linearly independent in $\mathbb{E}^{n+1}$. Conversely, suppose that $\left\{\left(a_{i}, 1\right)\right\}, i=0, \ldots, n$, is linearly independent in $\mathbb{E}^{n+1}$, i.e., $\sum_{i=1}^{n} \mu_{i}\left(a_{i}, 1\right)=0_{\mathbb{E}^{n+1}}$ only when $\mu_{i}=0, i=0, \ldots, n$. Therefore, $\sum_{i=0}^{n} \mu_{i} a_{i}=0_{\mathbb{E}^{n}}$ and $\sum_{i=0}^{n} \mu_{i}=0_{\mathbb{R}}$ occurs only when $\mu_{i}=0, i=0, \ldots, n$. Thus, $\left\{a_{i}\right\}, i=0, \ldots, n$, is affinely independent in $\mathbb{E}^{n}$, and the result follows.

\subsection{Pure state polytopes}

Definition 5.2 A pure state polytope is a polytope whose vertex description is given by a set of pure states, i.e., conv $\left\{\varrho_{i}\right\}$ where $\varrho_{i} \in \mathcal{M}_{d}$ such that $\operatorname{Tr} \varrho_{i}^{2}=1, \varrho_{i}=\varrho_{i}^{\dagger}, \varrho_{i} \geq 0$ and $\operatorname{Tr} \varrho_{i}=1$. A pure state sign permutation polytope is a polytope whose vertex set is constructed by applying the set of sign permutations on a pure state and admitting only those sign permutations that yield a valid pure quantum state.

Let $\varrho \in \mathcal{M}_{2}$ be a pure qubit state,

$$
\varrho=1 / 2\left(\begin{array}{cc}
1+P_{z} & P_{x}-i P_{y} \\
P_{x}-i P_{y} & 1-P_{z}
\end{array}\right)
$$

with $P_{x}{ }^{2}+P_{y}{ }^{2}+P_{z}{ }^{2}=1$ and $P_{x}, P_{y}, P_{z} \in \mathbb{R}$. The parametrization of $\varrho \in \mathcal{M}_{2}$ is given by $\left(P_{x}, P_{y}, P_{z}\right) \in \mathbb{R}^{3}$. Applying the set of sign permutations to the qubit parametrization $(1 / \sqrt{2}, 0,1 / \sqrt{2})$ and taking only those sign permutations that return valid pure quantum states yields the cuboctahedron of Figure 2 . 


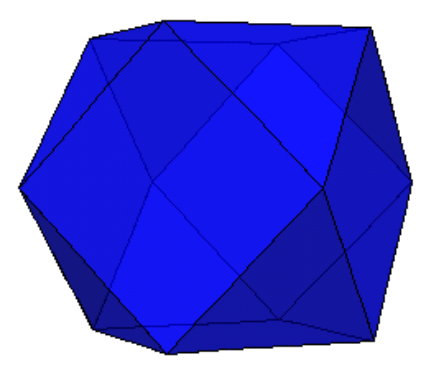

Figure 2. Qubit Cuboctahedron: sign permutation polytope with basis vector $(1 / \sqrt{2}, 0,1 / \sqrt{2})$. The cuboctahedron consists of 8 triangular faces, six square faces, 24 edges and 12 vertices.

For the construction of a quantum sign permutation polytope from a pure state $|\psi\rangle \in \mathcal{H}$, we enumerate the set of sign permutations on the Euclidean representative of density matrix $|\psi\rangle\langle\psi| \in \mathcal{M}_{d}$ and consider only those sign permutations $\pm \pi_{\text {pure }}$ for which $\pm \pi_{\text {pure }}(|\psi\rangle\langle\psi|)$ is again a pure state. For three-qubit systems, the general description of a pure state $|\psi\rangle$ is given by

$$
|\psi\rangle=\lambda_{0}|000\rangle+\lambda_{1} e^{\iota \theta}|100\rangle+\lambda_{2}|101\rangle+\lambda_{3}|110\rangle+\lambda_{4}|111\rangle
$$

where $\lambda_{i} \geq 0,0 \leq \theta \leq \pi$ and $\sum_{i} \lambda_{i}^{2}=1$ (Acín et al (2001)). Dür et al (2000) have shown that non-trivial tripartite entanglement of three qubit systems can be described in terms of two equivalence classes. In particular, a state in the system of three qubits possessing genuine tripartite entanglement may be a Greenberger-Horne-Zeilinger (GHZ) state for which a typical representative state is given by

$$
|\mathrm{GHZ}\rangle=1 / \sqrt{2}(|000\rangle+|111\rangle) .
$$

On the other hand, a three-qubit state may be in the $\mathrm{W}$ class of tripartite entangled states. A quantum state is said to be $\mathrm{W}$-type entangled if $\lambda_{4}=\theta=0$ in (19). A typical representative state for those states possessing $\mathrm{W}$-type entanglement is given by

$$
|W\rangle=1 / \sqrt{3}(|100\rangle+|010\rangle+|001\rangle) .
$$

As an example, we construct a quantum polytope of high dimensionality for some fixed affine space that possesses $\mathrm{W}$-type entanglement. For such a polytope, we considered a random three-qubit pure W-state $|W\rangle=0.758 \iota|0\rangle+(0.809-0.588 \iota)|2\rangle$ $+(0.809+0.588 \iota)|5\rangle+0.242|7\rangle$. Taking the set of all possible sign permutations on the parameterized vector, we construct the polytope vertex set by only including those sign permutations that correspond that valid W-type entangled pure quantum states. $\mathrm{W}$-type entanglement is ensured by showing a vanishing 3-tangle on the set of valid quantum states. The 3-tangle, $\tau_{3}$, is the measure most often utilized to distinguish between the GHZ- and W- class of three-qubit states (Coffman et al (2000)). For pure states, the 3-tangle is equivalent to the absolute value of a quantity known as Cayley's hyperdeterminant (Cayley (1845)), and is known to vanish for those states 
possessing W-type entanglement. In our example, there are $2^{4} 8 ! / 4 !=26880$ possible sign permutations for a general three-qubit W-type entangled state of which a total of 5376 pure points are returned as possessing W-type entanglement.

\subsection{Mixed state polytopes}

Definition 5.3 A mixed state polytope is a polytope whose vertex description is given by a set of mixed states, i.e., conv $\left\{\varrho_{i}\right\}$ where $\varrho_{i} \in \mathcal{M}_{d}$ such that $\operatorname{Tr} \varrho_{i}^{2}<1, \varrho_{i}=\varrho_{i}^{\dagger}, \varrho_{i} \geq 0$ and $\operatorname{Tr} \varrho_{i}=1$. A mixed state sign permutation polytope is a polytope whose vertex set is constructed by applying the set of sign permutations on a mixed state and admitting only those sign permutations that yield a valid quantum state.

An open problem of multipartite entanglement is the identification of the entanglement type for a given quantum state. Appealing to the theory of convex sets may help resolve the issue of multipartite entanglement identification whereby a state of unknown entanglement type is described as a convex combination of states of a fixed entanglement type. As noise has always to be considered for an experiment, we are interested in describing a volume (an $\epsilon$-ball) around the given state which is still of the same entanglement type. However, achieving a lower bound on the allowed $\epsilon$ environment requires knowledge about the facet set of a polytope of a fixed entanglement type. For $n$-dimensional convex polytopes consisting of a vertex set of order $m$, the set of hyperplanes can be of the order $m^{\lfloor n / 2\rfloor}$ (Matousek (2002)). In this instance, an $\epsilon$-ball argument to identify multipartite entanglement is rendered inefficient.

Let us now suppose that there exists a convex decomposition of a given state $\varrho \in \mathcal{M}_{d}$ in terms of states of a fixed entanglement type (Kampermann et al (2010)). We consider the construction of quantum sign permutation polytopes whose vertex set is now contained in the convex hull of the given convex decomposition. The volume of the constructed sign permutation polytope allows conclusions about the robustness of state identification. For efficiency in high dimensions, we consider the restriction of a general sign permutation polytope to that of the $n$-cross-polytope. The quantum polytope analysis proceeds in two parts. The initial part of the process is concerned with the construction of the regular $n$-cross-polytope. In a second algorithm we address the robustness of the identification process.

Algorithm 5.4 constructs a sign permutation polytope of edge length $\sqrt{2} \alpha, \alpha \in \mathbb{R}$, centered on a given state $\varrho \in \mathcal{M}_{d}$. The resulting polytope describes a convex hull of states of a certain entanglement type by ensuring that the vertices of the constructed quantum polytope be written as a convex composition of points from the initial convex set of states possessing a fixed entanglement type. We assume that the convex set input of algorithm 5.4 has order $m>d^{2}-1$. This is because we wish construct polytopes with non-zero volume centered on a mixed state in $\mathcal{M}_{d}$. 


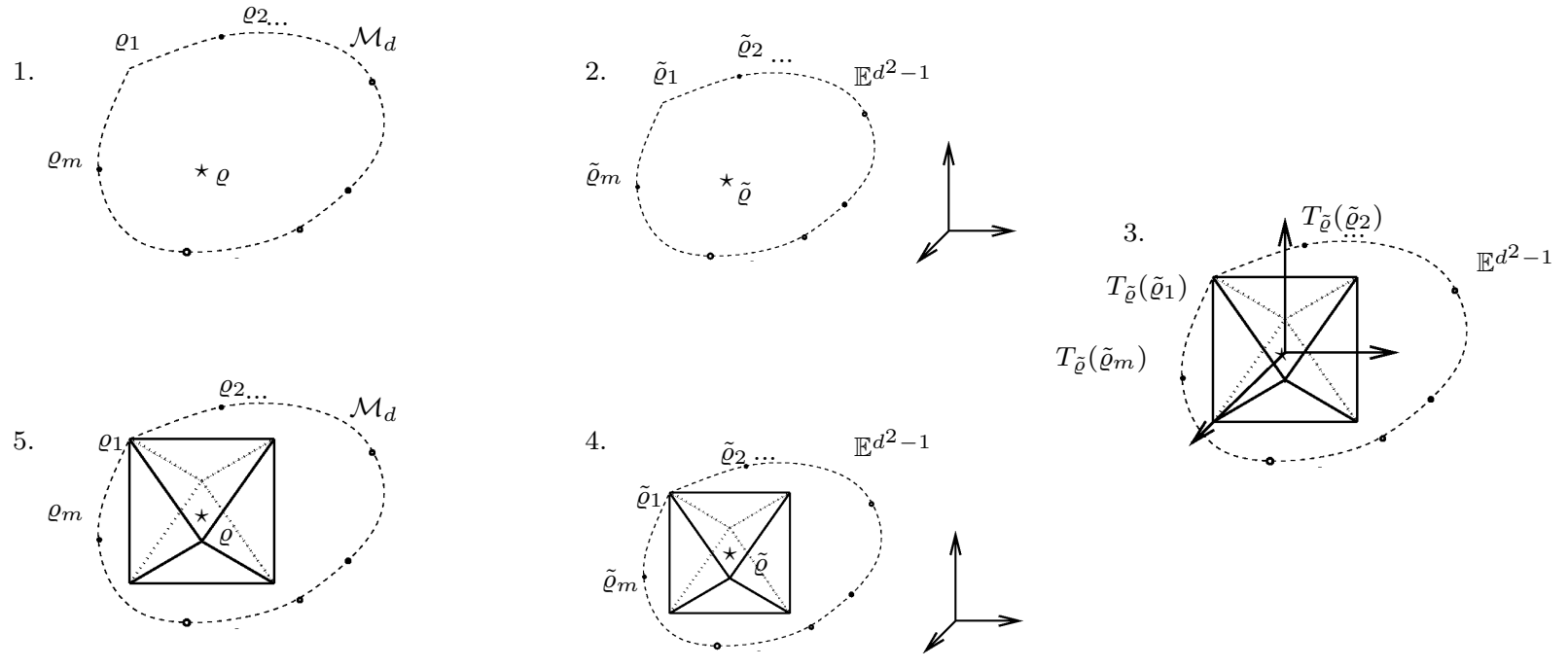

Figure 3. Constructing a quantum $\left(d^{2}-1\right)$-cross-polytope: $\mathcal{V}_{ \pm \pi(a)}, \pi \in S_{d^{2}-1}$ with basis vector $a=(\alpha, 0, \ldots, 0)$. 1 . illustrates the given state $\varrho$ as a convex combination of states of a fixed entanglement type. 2. 3. and 4. illustrate the construction of $\mathcal{V}_{ \pm \pi(a)}$ centered on $\tilde{\varrho}$ while 5 . shows a $\left(d^{2}-1\right)$-quantum-cross-polytope centered on the given state $\varrho \in \mathcal{M}_{d}$.

\section{Algorithm 5.4 Quantum polytope construction}

\section{Input:}

(i) Given state $\varrho \in \mathcal{M}_{d}$

(ii) Convex set of states of a fixed entanglement type, $\varrho_{i} \in \mathcal{M}_{d}$ for $i=1, \ldots, m$ and $m>d^{2}-1$, such that $\varrho=\sum_{i=1}^{m} \lambda_{i} \varrho_{i}, \lambda_{i} \geq 0$ and $\sum_{i=1}^{m} \lambda_{i}=1$. (Kampermann et al (2010))

Output: Quantum $\left(d^{2}-1\right)$-cross-polytope of a fixed entanglement type with edge $\overline{\text { length } \sqrt{2}} \alpha$ centered at $\varrho$, volume $(2 \alpha)^{d^{2}-1} /\left(d^{2}-1\right)$ ! and an inscribed sphere of radius $r_{\text {in }}=\alpha / \sqrt{d^{2}-1}$, see lemma 4.4 and lemma 4.5.

\section{Procedure:}

(i) Let $\tilde{\varrho} \in \mathbb{E}^{d^{2}-1}$ denote the Euclidean vector associated with $\varrho \in \mathcal{M}_{d}$. Let $\tilde{\varrho}_{i} \in$ $\mathbb{E}^{d^{2}-1}, i=1, \ldots, m$, be analogously defined.

(ii) Let $T_{\tilde{\varrho}}(a): a-\tilde{\varrho}, a \in \mathbb{E}^{d^{2}-1}$, be an affine transformation on $\mathbb{E}^{d^{2}-1}$. Let $\operatorname{conv}\left\{T_{\tilde{\varrho}}\left(\tilde{\varrho}_{i}\right), i=1, \ldots, m\right\}$ be the translate of $\operatorname{conv}\left\{\tilde{\varrho}_{i}, i=1, \ldots, m\right\}$ under $T_{\tilde{\varrho}}$.

(iii) For suitable $\alpha \in \mathbb{R}$, we ensure that the set of sign permutations of the vector $(\alpha, 0, \ldots, 0) \in \mathbb{E}_{+}^{d^{2}-1}$ is contained in the convex hull of $T_{\tilde{\varrho}}\left(\tilde{\varrho}_{i}\right), i=1, \ldots, m$.

(iv) Maximise $\alpha \in \mathbb{R}$ such that the set of $2\left(d^{2}-1\right)$ points $\left\{\pi( \pm \alpha, 0, \ldots, 0), \pi \in S_{d^{2}-1}\right\}$ are still contained in the convex hull of $T_{\tilde{\varrho}}\left(\tilde{\varrho}_{i}\right), i=1, \ldots, m$.

(v) Let $T_{\tilde{\varrho}}^{-1}(a)$ be an affine transformation on $\mathbb{E}^{d^{2}-1}$ given by $T_{\tilde{\varrho}}^{-1}(a): a+\tilde{\varrho}, a \in \mathbb{E}^{d^{2}-1}$. Then

$$
\operatorname{conv}\left\{T_{\tilde{\varrho}}^{-1}\left(\left\{\pi( \pm \alpha, 0, \ldots, 0), \pi \in S_{d^{2}-1}\right\}\right)\right\}
$$


is the $\left(d^{2}-1\right)$-dimensional cross-polytope with edge length $\sqrt{2} \alpha$ and volume $(2 \alpha)^{d^{2}-1} /\left(d^{2}-1\right)$ ! centered at $\tilde{\varrho}$ and contained in the convex hull conv $\left\{\tilde{\varrho}_{i}, i=\right.$ $1, \ldots, m\}$.

Step (i) of algorithm 5.4 establishes a correspondence between the set of density operators $\mathcal{M}_{d}$ and vectors in Euclidean space $\mathbb{E}^{d^{2}-1}$, see figure 3 (1. and 2.). The quantum states $\varrho$ and $\varrho_{i}$, for $i=1, \ldots, m$, are mapped to their corresponding representatives in $\mathbb{E}^{d^{2}-1}$. Step (ii) describes the translation transformation $T_{\tilde{\varrho}}$ that acts on the set of classical vectors $\tilde{\varrho}$ and $\tilde{\varrho}_{i}$, for $i=1, \ldots, m$. Under $T_{\tilde{\varrho}}$, the vector $\tilde{\varrho} \in \mathbb{E}^{d^{2}-1}$ is mapped to the $o \in \mathbb{E}^{d^{2}-1}$. Correspondingly, the set $\tilde{\varrho}_{i}$, for $i=1, \ldots, m$, is mapped to a surrounding neighbourhood of the origin. Step (iii) then constructs a sign permutation polytope with edge length $\sqrt{2} \alpha$ centered at origin for the basis vector $a=(\alpha, 0, \ldots, 0) \in \mathbb{E}_{+}^{d^{2}-1}$, see figure 3 (3.). Step (iv) utilizes a divide and conquer algorithm (O' Rourke (1998)) to ensure that the value $\alpha \in \mathbb{R}$ in the basis vector induces the polytope of maximum volume. By lemma 4.5, the constructed sign permutation polytope has volume $(2 \alpha)^{d^{2}-1} /\left(d^{2}-1\right)$ !. Since this polytope is defined by taking the sign permutations of the vector $(\alpha, 0, \ldots, 0) \in \mathbb{E}_{+}^{d^{2}-1}$, the construction yields the regular $\left(d^{2}-1\right)$-dimensional cross-polytope. Step (v) implements the translation transformation $T_{\varrho}^{-1}$ on the set of vertices of the constructed cross-polytope. Since a translate of a convex polytope is again a convex polytope, a consequence of result $x+\operatorname{conv} M=\operatorname{conv}(x+M)(\operatorname{Br} \varnothing$ ndsted $(1983))$, for $x \in \mathbb{E}^{n}$ and $M \subset \mathbb{E}^{n}$, it follows that $\operatorname{conv}\left\{T_{\tilde{\varrho}}^{-1}\left(\left\{\pi( \pm \alpha, 0, \ldots, 0), \pi \in S_{d^{2}-1}\right\}\right)\right\}$ is a regular cross-polytope centered at $\tilde{\varrho}$ with edge length $\sqrt{2} \alpha$ and volume $(2 \alpha)^{d^{2}-1} /\left(d^{2}-1\right)$ !, see figure 3 (4.). Since any $\left(d^{2}-1\right)$ dimensional affine space is isomorphic to the Euclidean space $\mathbb{E}^{d^{2}-1}$, the constructed polytope yields a desired quantum polytope in $\mathcal{M}_{d}$ with respect to the Hilbert-Schmidt norm, see figure 3 (5.).

The next stage in the process addresses the question of robustness of multipartite entanglement identification. Algorithm 5.6 makes use of the weak majorization criterion to determine the points $\varrho^{\prime} \in \mathcal{M}_{d}$ in the convex hull of a constructed sign permutation polytope. The set of points $\varrho^{\prime} \in \mathcal{M}_{d}$ can be represented as the state $\varrho \in \mathcal{M}_{d}$ plus noise in the quantum system. We make use of the following result from Życzkowski and Sommers (2003).

Theorem 5.5 (Życzkowski and Sommers (2003)) The volume of the $\left(d^{2}-1\right)$ dimensional set of states $\mathcal{M}_{d}$ with respect to the Hilbert-Schmidt measure is given by

$$
\mathrm{Vol}_{\mathrm{HS}}\left(\mathcal{M}_{d}\right)=\sqrt{d} \frac{\pi^{d(d-1) / 2}}{2^{(d-1) / 2}} \frac{\Gamma(1) \ldots \Gamma(d)}{\Gamma\left(d^{2}\right)}
$$

where $\Gamma(\cdot)$ is the Euler gamma function.

\section{Algorithm 5.6 On the robustness of state identification Input:}

(i) Cross polytope conv $\left\{\pi( \pm \alpha, 0, \ldots, 0), \pi \in S_{d^{2}-1}\right\}$ of edge length $\sqrt{2} \alpha$ and volume $(2 \alpha)^{d^{2}-1} / d^{2}-1$ ! associated with a quantum polytope of a fixed entanglement type. 


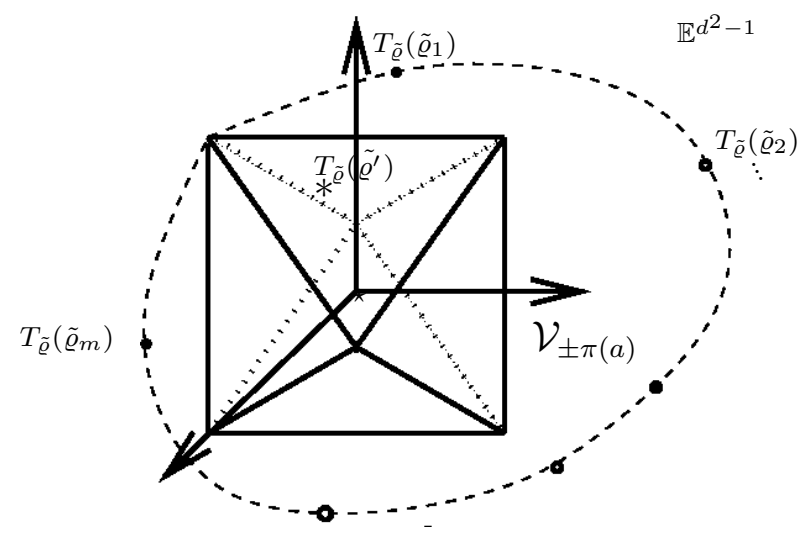

Figure 4. Translate of an arbitrary state $\varrho^{\prime}, T_{\tilde{\varrho}}\left(\tilde{\varrho^{\prime}}\right)$, contained in the convex hull of the $\left(d^{2}-1\right)$-cross-polytope $\mathcal{V}_{ \pm \pi(a)}, \pi \in S_{d^{2}-1}$ with basis vector $a=(\alpha, 0, \ldots, 0)$.

(ii) Given state $\varrho \in \mathcal{M}_{d}$.

(iii) An arbitrary state $\varrho^{\prime} \in \mathcal{M}_{d}$.

Output: The fraction of the volume of $\mathcal{M}_{d}$ describing the set of all states in the constructed sign permutation polytope possessing an entanglement type comparable to the state $\rho \in \mathcal{M}_{d}$.

\section{Procedure:}

(i) Let $\tilde{\varrho}^{\prime} \in \mathbb{E}^{d^{2}-1}$ denote the Euclidean vector associated with an arbitrary state $\varrho^{\prime} \in \mathcal{M}_{d}$

(ii) Let $T_{\tilde{\varrho}}\left(\tilde{\varrho}^{\prime}\right)$ be the image of $\tilde{\varrho}^{\prime}$ under the mapping $T_{\tilde{\varrho}}(a): a-\tilde{\varrho}, a \in \mathbb{E}^{d^{2}-1}$.

(iii) Consider the positive cone $\mathbb{E}_{+}^{d^{2}-1}:=\left\{\left(\alpha_{1}, \ldots, \alpha_{d^{2}-1}\right) \in \mathbb{E}^{d^{2}-1} \mid \alpha_{i} \geq 0\right\}$. For suitable sign changes, let $T_{+}\left(\tilde{\varrho}^{\prime}\right)$ be the image of $T_{\tilde{\varrho}}\left(\tilde{\varrho}^{\prime}\right)$ in the positive cone $\mathbb{E}_{+}^{d^{2}-1}$.

(iv) Implement corollary 4.1: Letting $\mathcal{V}_{ \pm \pi(a)}$ denote the cross-polytope $\operatorname{conv}\{\pi( \pm \alpha, 0, \ldots, 0)$, $\left.\pi \in S_{d^{2}-1}\right\}$ of edge length $\sqrt{2} \alpha$. Then

$$
T_{+\tilde{\varrho}}\left(\tilde{\varrho^{\prime}}\right) \in \mathcal{V}_{ \pm \pi(\alpha)} \Leftrightarrow T_{+\tilde{\varrho}}\left(\tilde{\varrho^{\prime}}\right) \prec_{w} \alpha .
$$

(v) If $T_{+\tilde{\varrho}}\left(\tilde{\varrho^{\prime}}\right) \prec_{w} \alpha$ then $T_{+\tilde{\varrho}}\left(\tilde{\varrho}^{\prime}\right)$ is contained in sign permutation polytope $\mathcal{V}_{ \pm \pi(\alpha)}$. Consequently, $T_{+}\left(\tilde{\varrho}\left(\tilde{\varrho}^{\prime}\right)\right.$ represents a state in the

$$
\frac{2^{(2 d+3)(d-1) / 2} \alpha^{d^{2}-1}}{\sqrt{d} \pi^{d(d-1) / 2} \Gamma(1) \ldots \Gamma(d)}
$$

fraction of all states contained in sign permutation polytope $\mathcal{V}_{ \pm \pi(\alpha)}$ which possesses an entanglement type comparable to state $\rho \in \mathcal{M}_{d}$.

Step (i) of algorithm 5.6 considers a given arbitrary state $\varrho^{\prime} \in \mathcal{M}_{d}$ to describe the set of states differing from $\varrho \in \mathcal{M}_{d}$. By analyzing the classical representation of $\varrho^{\prime} \in \mathcal{M}_{d}$, a characterization of quantum states possessing an entanglement type comparable to $\mathcal{V}_{ \pm \pi(\alpha)}$ can be achieved. Step (i) begins by evaluating the classical representation of $\varrho^{\prime} ; \tilde{\varrho}^{\prime} \in \mathbb{E}^{d^{2}-1}$. Step (ii) implements the translation transformation $T_{\tilde{\varrho}}(a)$ on 
$\tilde{\varrho}^{\prime} \in \mathbb{E}^{d^{2}-1}$ thus translating $\tilde{\varrho}^{\prime} \in \mathbb{E}^{d^{2}-1}$ to a neighbourhood of the cross-polytope $\mathcal{V}_{ \pm \pi(\alpha)}=\operatorname{conv}\left\{\pi( \pm \alpha, 0, \ldots, 0), \pi \in S_{d^{2}-1}\right\}$. Step (iii) prepares the point $\tilde{\varrho}^{\prime} \in \mathbb{E}^{d^{2}-1}$ for the weak majorization criterion of corollary 4.1 by placing $\tilde{\varrho}^{\prime} \in \mathbb{E}^{d^{2}-1}$ in the positive cone $\mathbb{E}_{+}^{d^{2}-1}$ for suitable sign changes. Step (iv) then implements the weak majorization criterion for point inclusion in a sign permutation polytope. If $T_{+\tilde{\varrho}}\left(\tilde{\varrho^{\prime}}\right) \prec_{w} \alpha$ then noting $T_{\tilde{\varrho}}\left(\tilde{\varrho}^{\prime}\right) \prec_{w} T_{+\tilde{\varrho}}\left(\tilde{\varrho}^{\prime}\right)$, it follows by the transitive property of weak majorization that $T_{\tilde{\varrho}}\left(\tilde{\varrho^{\prime}}\right) \prec_{w} \alpha$, see figure 4 . Consequently, $T_{\tilde{\varrho}}\left(\tilde{\varrho}^{\prime}\right) \in \mathcal{V}_{ \pm \pi(a)}$. Finally, step (v) returns the fraction of states of $\mathcal{M}_{d}$ contained in the quantum cross-polytope $\mathcal{V}_{ \pm \pi(\alpha)}$ centered on the state $\varrho \in \mathcal{M}_{d}$. This fraction is given as ratio of the volume of the quantum cross-polytope $\mathcal{V}_{ \pm \pi(\alpha)}$ to the volume of the set of all density matrices (Życzkowski and Sommers (2003)).

Algorithm 5.6 introduced the fraction of all states of $\mathcal{M}_{d}$ contained in a quantum sign permutation polytope possessing an entanglement type equivalent to the entanglement type of the state centered at the given quantum polytope. This fraction was established in terms of the ratio of Hilbert-Schmidt volumes. However, a Euclidean$\epsilon$-ball argument describing the distance between a given state and a set of states of comparable entanglement type may also be considered. By lemma 4.5, the insphere of the constructed quantum polytope $\operatorname{conv}\left\{\pi( \pm \alpha, 0, \ldots, 0), \pi \in S_{d^{2}-1}\right\}$ given in algorithm 5.4 is of radius $\alpha / \sqrt{d^{2}-1}$. Letting $\epsilon=\alpha / \sqrt{d^{2}-1}$, the set of states $\varrho^{\prime} \in \mathcal{M}_{d}$ contained in $\epsilon$-ball possessing an entanglement type comparable to that of the state $\varrho \in \mathcal{M}_{d}$ is given by the Hilbert-Schmidt distance

$$
\left\|\varrho^{\prime}-\varrho\right\|_{\mathrm{HS}} \leq \alpha / \sqrt{d^{2}-1} .
$$

Now we note that the volume of an $n$-dimensional sphere of radius $r$ is $V_{n}=S_{n-1} r^{n} / n$ with $S_{n-1}=2 \pi^{n / 2} / \Gamma\left(\frac{n}{2}\right)$ denoting the hyper-surface area of an $n$-sphere of unit radius, and $\Gamma(\cdot)$ denoting the Gamma function (Bengtsson and Życzkowski (2006)). For radius $r_{i n}=\alpha / \sqrt{d^{2}-1}$, the $\left(d^{2}-1\right)$-dimensional insphere of the cross-polytope of edge length $\sqrt{2} \alpha$ has volume

$$
V_{d^{2}-1}=\frac{2 \pi^{\left(d^{2}-1\right) / 2} \alpha^{d^{2}-1}}{\Gamma\left(\frac{d^{2}-1}{2}\right)\left(d^{2}-1\right)^{d^{2}+1}} .
$$

On the other hand, the volume of the $\left(d^{2}-1\right)$-dimensional cross-polytope of edge length $\sqrt{2} \alpha$ is $(2 \alpha)^{d^{2}-1} /\left(d^{2}-1\right)$ !. Comparing these volumes yields an estimate that the $\left(d^{2}-1\right)$-dimensional insphere of radius $\alpha / \sqrt{d^{2}-1}$ is approximately $(\pi / 4)^{\left(d^{2}-1\right) / 2}$ that of its respective cross-polytope. Consequently, while the characterization of arbitrary states with respect to polytope construction herein which possess an entanglement type comparable to the given state $\varrho \in \mathcal{M}_{d}$ is readily obtainable by both weak majorization and Euclidean norm calculations, arguing robustness of identification in terms of volume ratios between quantum sign permutation polytopes and the space of density matrices may be of some merit.

Finally, the lower bound on the fraction of states introduced in algorithm 5.6 may be improved by considering the construction of the sign permutation polytope $\mathcal{V}_{ \pm \pi(\alpha)}$ with $a=\left(\alpha_{1}, \ldots, \alpha_{m}, 0_{1}, \ldots, 0_{d^{2}-1-m}\right), m \leq d^{2}-1$, denoting a multiset of $k$ distinct 
elements of multiplicity $m_{i}(1 \leq i \leq k)$. This approach is more costly and requires that $2^{m} n ! /\left(m_{1} ! \ldots m_{k-1} !(n-m) !\right)$ vertices be evaluated as convex combinations of an initially given convex set of density matrices with a fixed entanglement type. Furthermore, the resulting polytope structure loses regularity exhibited by the $n$-cube and $n$-crosspolytope thereby making volume calculation difficult.

\section{Conclusion}

We discussed the construction of $n$-dimensional polytopes of degree $n$ in the $n$ dimensional Euclidean space $\mathbb{E}^{n}$ called sign permutation polytopes, and presented a necessary and sufficient condition that characterizes the points of these polytopes. We related the construction of sign permutation polytopes in Euclidean space to the space of density matrices. As an application of such quantum polytopes, we considered the robustness of identifying those states of a fixed entanglement type. This process proceeded in two stages, the first stage concerned the construction of a convex polytope with a vertex set of a fixed type entanglement. The second stage characterized via weak majorization the fraction of all quantum states contained in the convex hull of a constructed convex polytope which possess an entanglement type comparable to the polytope's center state.

\section{Acknowledgments}

The authors wish to thank David Gross and Eshan Momtahan for helpful comments and suggestions. We gratefully thank DFG for financial support.

\section{Appendix A}

Proof of lemma 3.4. Let us suppose otherwise. Then for $x \in \mathcal{V}_{a}$ and $y \in \mathcal{V}_{b}$ there is a hyperplane $H_{u, \alpha}$ such that

$$
\langle x, u\rangle<\alpha \leq\langle y, u\rangle \text {. }
$$

Since $\sum_{k \leq n} y_{k}=\sum_{k \leq n} \beta_{k}$, we may take the hyperplane $H_{1_{n}, \sum_{k \leq n} y_{k}}, 1_{n}=(1, \ldots, 1) \in$ $\mathbb{E}^{n}$, to be the representative for the set of hyperplanes $H_{u, \alpha}$ that place $\mathcal{V}_{b}$ in one of the closed halfspaces of $\mathbb{E}^{n}$, see Appendix B. In particular, $\mathcal{V}_{b}$ is in the halfspace $H_{1_{n}, \sum_{k \leq n} y_{k}}^{+}$ that satisfies $\sum_{k \leq n} y_{k} \leq\left\langle y, 1_{n}\right\rangle$. Now $\left\langle x, 1_{n}\right\rangle=\sum_{k \leq n} x_{k}$. Consider the sum $\sum_{k \leq n} x_{k}$. For $x \in \mathcal{V}_{a}$ there are non-negative scalars $\lambda_{i}, i=1, \ldots, m$ with $m \leq n$ ! such that $\sum_{i \leq m} \lambda_{i}=1$ and $x=\sum_{i \leq m} \lambda_{i} a_{i}$. Therefore, we have it that

$$
\sum_{k \leq n} x_{k}=\sum_{k \leq n} \sum_{i \leq m} \lambda_{i} \alpha_{i k}=\sum_{i \leq m} \lambda_{i} \sum_{k \leq n} \alpha_{i k}=\sum_{i \leq m} \lambda_{i} \sum_{k \leq n} a_{k},
$$

since $\sum_{k \leq n} \alpha_{i k}=\sum_{k \leq n} \alpha_{k}$ for $i=1, \ldots, m$. Furthermore, since $\sum_{k \leq n} \alpha_{k}=\sum_{k \leq n} \beta_{k}$, we have $\alpha_{j}=\sum_{l \leq n} \beta_{l}-\sum_{\substack{l \leq n \\ l \neq j}} \alpha_{l}$ for $j \in\{1, \ldots, n\}$. It follows that equation A.2 may 


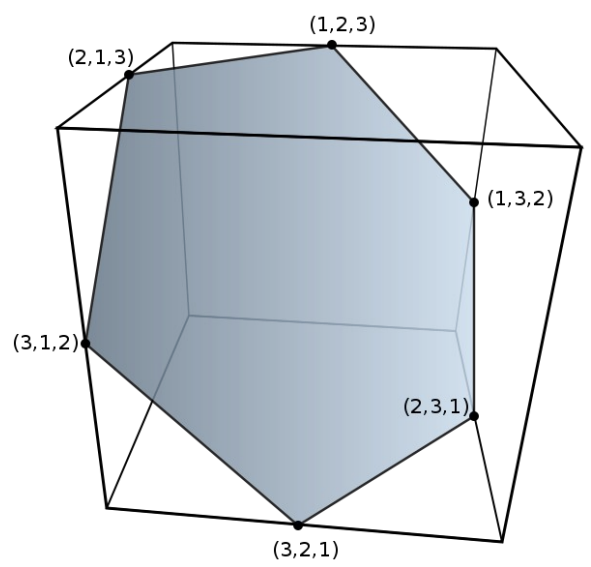

Figure B1. Permutahedron: $\mathcal{V}_{a}, a=(1,2,3)$

be written as

$$
\sum_{i \leq m} \lambda_{i}\left(\sum_{\substack{k \leq n \\ k \neq j}} \alpha_{k}+\sum_{l \leq n} \beta_{l}-\sum_{\substack{l \leq n \\ l \neq j}} \alpha_{l}\right)
$$

for $j \in\{1, \ldots, d\}$. Simplifying, we write equation (A.3) as $\sum_{i \leq m} \lambda_{i} \sum_{l \leq n} \beta_{l}$. Since there are numbers $\mu_{i} \geq 0$ satisfying $\sum_{i \leq m^{\prime}} \mu_{i}=1$, we then have it that $\sum_{i \leq m} \lambda_{i} \sum_{l \leq n} \beta_{l}=$ $\sum_{i \leq m^{\prime}} \mu_{i} \sum_{l \leq n} \beta_{l}$. Moreover, since $\sum_{k \leq n} \beta_{k}=\sum_{k \leq n} \beta_{i k}$, for $i=1, \ldots, m^{\prime}$, it follows that $\sum_{i \leq m^{\prime}} \mu_{i} \sum_{l \leq n} \beta_{l}$ may be given by

$$
\sum_{i \leq m^{\prime}} \mu_{i} \sum_{l \leq n} \beta_{l}=\sum_{l \leq n} \sum_{i \leq m^{\prime}} \mu_{i} \beta_{l}=\sum_{l \leq n} \sum_{i \leq m^{\prime}} \mu_{i} \beta_{i l}=\sum_{l \leq n} y_{l} .
$$

Therefore, $\left\langle x, 1_{n}\right\rangle=\left\langle y, 1_{n}\right\rangle$, and this contradicts the initial assumption that the convex hulls $\mathcal{V}_{a}$ and $\mathcal{V}_{b}$ can be properly separated. This completes the proof.

\section{Appendix B}

We construct the hyperplane $H_{u, \alpha}$ with the property $H_{u, \alpha} \cap \mathcal{V}_{a} \neq \emptyset$.

Example 1: Constructing hyperplanes for the permutahedron $\mathcal{V}_{\mathrm{a}}$ with $\mathrm{a}=(1,2,3)$, see figure B1.

Taking the points $(2,1,3)$ and $(2,3,1)$, we have it that the parametric equation of hyperplane (line) containing these points is given by $H_{1}:(0,2,-2) s+(2,3,1)$, for $s \in \mathbb{E}$. For any $z \in H_{1}$, we may write $\mathrm{z}$ componentwise as $z_{1}=2, z_{2}=2 s+3, z_{3}=-2 s+1$, $s \in \mathbb{E}$. Now since a hyperplane may be written in the form

$$
H_{u, \alpha}=\left\{z \in \mathbb{E}^{d} \mid\langle z, u\rangle=\alpha\right\}
$$

for $\mathrm{u} \in \mathbb{E}^{d} \backslash\{0\}$, and $\alpha \in \mathbb{R}^{d}$, we may rewrite $H_{1}$ in terms of the $H_{u, \alpha}$ form. We put $\alpha=\sum a_{k}=6$. Then $2 u_{1}+(2 s+3) u_{2}+(-2 s+1) u_{3}=r$. In particular, we have it that $H_{u, 6}$ is constrained by $2 u_{1}+4 u_{2}=6$ with $u_{2}=u_{3}$. We determine the remaining hyperplanes in a similar fashion. Proceeding anticlockwise, $H_{2}=(1,-1,0) s+(3,2,1)$, for $s \in \mathbb{E}$. Rewriting $H_{2}$ in terms of an $H_{\mathrm{u}, 6}$ expression, the candidate vector $\mathrm{u} \in \mathbb{E}^{3}$ is constrained 
by the equation $5 u_{1}+u_{3}=6$ with $u_{1}=u_{2}$. The hyperplane $H_{3}=(0,-1,1) s+(3,1,2)$, for $s \in \mathbb{E}$, rewriting $H_{3}$, the candidate vector $u \in \mathbb{E}^{3}$ is constrained by the equation $3 u_{1}+3 u_{2}=6$ with $u_{2}=u_{3}$. The hyperplane $H_{4}=(-2,2,0) s+(1,3,2)$, for $s \in \mathbb{E}$, rewriting $H_{4}$, the candidate vector $u \in \mathbb{E}^{3}$ is constrained by the equation $4 u_{1}+2 u_{3}=6$ with $u_{1}=u_{2}$. The hyperplane $H_{5}=(0,1,-1) s+(1,3,2)$, for $s \in \mathbb{E}$, rewriting $H_{5}$, the candidate vector $u \in \mathbb{E}^{3}$ is constrained by the equation $u_{1}+5 u_{2}=6$ with $u_{2}=u_{3}$. Finally, the hyperplane $H_{6}=(-1,1,0) s+(1,2,3)$, for $s \in \mathbb{E}$ and rewriting $H_{6}$ in the form $H_{u, 6}$, the candidate vector $u \in \mathbb{E}^{3}$ is constrained by the equation $3 u_{1}+3 u_{3}=6$ with $u_{1}=u_{2}$. Note that while each evaluated hyperplane has infinitely many solutions, a candidate that satisfies each of the particular constraints is the vector with components all equal to 1 . Of course, since the direction vectors in each of the parametric expressions are permutations of each other then a candidate vector $u \in \mathbb{E}^{3}$ which satisfies all of the above constraints is precisely that vector which, under permutation of indices corresponding to the direction vectors, generates a solution vector $u^{\prime} \in \mathbb{E}^{3}$ for all hyperplane $H_{u^{\prime}, 6}$. The only such candidate is the vector $u=(1,1,1)$.

Example 2: Constructing hyperplanes for $\mathcal{V}_{a}, a=(1,2, \ldots, n)$.

Continuing in the manner of above, we seek a candidate hyperplane for the set of all hyperplanes bounding $\mathcal{V}_{a}$ entirely in a closed halfspace. We claim the hyperplane given by $H_{1_{n}, \frac{n(n+1)}{2}}, 1_{n}=(1, \ldots, 1) \in \mathbb{E}^{n}$, is such a candidate. Taking the vector $a=(1,2, \ldots, n) \in \mathbb{E}^{n}$, the associated permutahedron $\mathcal{V}_{a}$ is defined as the convex hull of all vertices obtained by permuting the coordinates of the vector $a$. Since the set of vertices connected by and edge to the vector $a$ are exactly those permutations of vector $a$ that differ by an adjacent transposition, we have it that the set of vertices connected by an edge to vertex $a$ is the set $\{(1,2, \ldots, n-2, n, n-1)$, $(1,2, \ldots, n-1, n-2, n), \ldots,(2,1, \ldots, n)\}$. Taking the vertex $a \in \mathbb{E}^{n}$ together with any $n-2$ vertices of the connecting set, of which there are $n-1$ possible choices, determine a $(n-2)$-dimensional hyperplane. Fix the hyperplane $H_{1}$ to contain the set of vertices connected by an edge to the vector $a \in \mathbb{E}^{n}$ that exclude the last vertex $(2,1,3, \ldots, n)$. The parametric equation for the hyperplane $H_{1}$ is written in terms of the directions vectors $\{(0, \ldots, 0,1,-1),(0, \ldots, 0,1,-1,0), \ldots,(0,1,-1,0 \ldots, 0)\}$. Each direction vector is represented as the difference between the vertex $a$ and an element of the set $\{(1,2, \ldots, n-2, n, n-1),(1,2, \ldots, n-1, n-2, n), \ldots,(1,3,2, \ldots, n)\}$. Rewriting $H_{1}$ in the $H_{u, \alpha}$ form with $\alpha=\frac{n(n+1)}{2}$, we have it that the candidate vector $u \in \mathbb{E}^{n}$ is constrained by the equation $u_{1}+\left(\frac{n(n+1)}{2}-1\right) u_{2}=\frac{n(n+1)}{2}$ with $u_{2}=u_{3}=\ldots=u_{n}$. Next, the hyperplane $H_{2}$ determined by the vertex $a \in \mathbb{E}^{n}$ and its set of connecting vertices that excludes the vertex $(1,3,2,4, \ldots, n)$ has its parametric expression written as linear combination of the set of direction vectors $\{(0, \ldots, 0,1,-1),(0, \ldots, 0,1,-1,0), \ldots,(0,0,1,-1,0 \ldots, 0),(1,-1,0, \ldots, 0)\}$. Writing the parametric equation of the hyperplane $H_{2}$ in terms of the $H_{u, \frac{n(n+1)}{2}}$ form, we have the candidate vector $u \in \mathbb{E}^{n}$ constrained by $u_{1}+\left(\frac{n(n+1)}{2}-1\right) u_{3}=\frac{n(n+1)}{2}$ with $u_{1}=u_{2}$ and 
$u_{3}=u_{4}=\ldots=u_{n}$. In a similar manner, the remaining $n-3$ hyperplanes containing the vertex $a$ can be formed. Solving for the vector $u \in \mathbb{E}^{n}$ that determines the hyperplane $H_{u, \frac{n(n+1)}{2}}$ associated with $H_{1}$ and $H_{2}$ yields the vector $1_{n}=(1, \ldots, 1) \in \mathbb{E}^{n}$. It follows that $\stackrel{2}{H}_{1_{n}, \frac{n(n+1)}{2}}$ may be taken as the representative for the set of hyperplanes containing vertex $a \in \mathbb{E}^{n}$. Furthermore, since all vertices $a^{\prime} \in \mathbb{E}^{n}$ of the permutahedron $\mathcal{V}_{a} \subset \mathbb{E}^{n}$ are permutations of the vertex $a$, for $\pi_{a^{\prime}} \in \mathcal{S}_{n}$, then the set of hyperplanes containing the point $a^{\prime} \in \mathbb{E}^{n}$ can be rewritten in terms of a hyperplane $H_{u, \frac{n(n+1)}{2}}$ for some candidate vector $u \in \mathbb{E}^{d}$. For such a candidate $u \in \mathbb{E}^{n}$ and permutation $\pi_{a^{\prime}}^{-1} \in \mathcal{S}_{n}, H_{\pi_{a^{\prime}}^{-1}(u), \frac{n(n+1)}{2}}$ is a representative hyperplane for the set of hyperplane containing the vertex $a \in \mathbb{E}^{n}$. Consequently, $H_{\pi_{a^{\prime}}^{-1}(u), \frac{n(n+1)}{2}}=H_{1_{n}, \frac{n(n+1)}{2}}$. In particular, $\pi_{a^{\prime}}^{-1}(u)=1$. Since the vector with all components equal to 1 is invariant under permutations of the indices, it follows that $H_{1, \frac{n(n+1)}{2}}$ may be taken as a representative for the set of all hyperplanes bounding $\mathcal{V}_{a}$ entirely in a closed halfspace with the property that $H_{z, \alpha} \cap \mathcal{V}_{a} \neq \emptyset$.

\section{References}

Acín A, Andrianov A, Jane E and Tarrach R 2001 Three-qubit pure-state canonical forms J. Phys. A: Math. Gen. 34 6725-39

Bishop E and Bridges D 1985 Constructive Analysis (Berlin: Springer) (1985)

Bengtsson I and Życzkowski K 2006 Geometry of quantum states (New York: Cambridge University Press)

Bennett C H, Bernstein H J, Popescu S and Schumacher B 1996 Concentrating partial entanglement by local operations Phys. Rev. A 53 2046-52

Brøndsted 1983 An introduction of convex polytopes (New York: Springer-Verlag)

Cayley A 1845 On the theory of linear transformations Cambridge Math. J. 4 193-209

Coffman V, Kundu J and Wootters W K 2000 Multipartite pure-state entanglement and the generalized GHZ states Phys. Rev. A 61052306

Coxeter H S M 1969 Introduction to Geometry 2nd ed. (New York: John Wiley \& Sons, Inc.)

Coxeter H S M 1948 Regular Polytopes (London: Methuen \& Co. Ltd.)

Dür W, Vidal G and Cirac J I 2000 Three qubits can be entangled in two inequivalent ways Phys. Rev. A 62062314

Gaiha P and Gupta S K 1977 Adjacent vertices on a permutohedron SIAM J. Appl. Math. 32 323-327

Grünbaum 2003 Convex polytopes 2nd ed. (New York: Springer-Verlag)

Hardy G H, Littlewood J E and Pólya G 1934 Inequalities (New York: Cambridge University Press)

Kampermann K, Gühne O, Wilmott C and Bruss D 2010 unpublished

Markus 1964 Eigenvalues and singular values of sum and product of linear operators Russian Math. Surveys 19 91-120

Marshall A W and Olkin I 1979 Inequalities: theory of majorization and its applications (New York: Academic Press)

Matousek J 2002 Lectures on Discrete Geometry (New York: Springer-Verlag)

Mirsky L 1959 On a convex set of matrices Arch. Math. 10 88-92

O' Rourke J 1998 Computational Geometry in C (New York: Cambridge University Press)

Vidal G, Dür W and Cirac J I 2000 Reversible combination of inequivalent kinds of multipartite entanglement Phys. Rev. Lett. 85 658-61

Rado R 1952 An equality J. London Math. Soc. 27 1-6

Ziegler G M 1995 Lectures on polytopes (Berlin: Springer-Verlag) 
Życzkowski K and Sommers H -J 2003 Hilbert schmidt volume of the set of mixed quantum states $J$. Phys. A 36 10115-30 